\title{
Scalp Psoriasis: A Brief Overview
}

\section{Meghana Madhukar Phiske*}

Department of Dermatology, L.T.M.M.C and General Hospital, Sion, India

${ }^{*}$ Corresponding author: Meghana Madhukar Phiske, Department of Dermatology, L.T.M.M.C and General Hospital, Sion, India, Residential address: N-17, Sector -7, Vashi, Navi Mumbai, India, Tel: 9819030429; E-mail: meghanaphiske@rediffmail.com

Received date: June 10, 2015; Accepted date: June 27, 2016; Published date: July 4, 2016

Copyright: () 2016 Phiske MM. This is an open-access article distributed under the terms of the Creative Commons Attribution License, which permits unrestricted use, distribution, and reproduction in any medium, provided the original author and source are credited.

\section{Introduction}

Psoriasis vulgaris is one of the common chronic skin diseases, which can affect any area of the skin, but scalp is one of the most frequently involved [1]. Although the head represents only $10 \%$ of the body's surface area, the consequences of scalp psoriasis are disproportionate to the area, as it can be seriously debilitating [2]. Scalp psoriasis is characterized by sharply demarcated erythematosquamous lesions with silver-white scaling [3]. It is a frequent expression of the common skin disease with scaling and itching being the two major complaints [4].

\section{Epidemiology}

Psoriasis vulgaris affects $1-3 \%$ of the general population [5], with the scalp being the most common site of involvement, at the onset and throughout the course of the disease [6]. In one study of 1220 patients with psoriasis, the scalp was described as the first site of onset in $25 \%$ [7].

Data from Vietnam, Malaysia, Philippines and Taiwan indicate that between $1.4 \%$ and $2.7 \%$ of patients have psoriasis and among these, between $75 \%$ and $90 \%$ have scalp involvement. Malaysian Psoriasis Registry (MPR) also reported scalp involvement in $78.8 \%$ of patients with psoriasis. This data reveals that the rate of scalp involvement in Asia is similar to or higher than in Western European populations, where it is between $50 \%$ and $80 \%$. Prevalence of scalp psoriasis is $1.5 \%$ to $2 \%$ in northwestern Europe [6]. Scalp involvement is reported in 47 $88.9 \%$ of all children with psoriasis [8].

Among juvenile plaque psoriasis patients from eight geographically diverse United States dermatology clinics, $79 \%$ had a history of scalp involvement [9].

\section{Etiopathogenesis}

The exact etiology of scalp psoriasis is unknown, the condition being complex, resembling or coexisting with seborrhoeic dermatitis. It can be associated with early or late-onset psoriasis vulgaris, guttate psoriasis, pustular psoriasis erythroderma or psoriatic arthritis [6].

\section{Association with Malassezia yeasts and Seborrhoeic dermatitis}

Overgrowth of the scalp with Malassezia species is a well-known feature of scalp psoriasis (malassezia are found in areas rich in sebaceous glands like scalp) [1]. The number of Malassezia spp. found indicate that Malassezia yeasts may be involved in the pathogenesis of scalp psoriasis and that scalp psoriasis may be due to pityrosporal colonization [6,10]. Gomez-Moyano et al. found that during periods of exacerbation the presence of Malassezia particularly $M$. globosa, are increased. Prohić found significant difference in distribution of Malassezia species between psoriatic and healthy scalp and in the distribution of Malassezia species according to the severity of the scalp involvement $[11,12]$. It is also thought that psoriasis may develop from seborrhoeic dermatitis due to a Koebner response to Malassezia yeast colonization. The clinical overlap of scalp psoriasis and seborrhoeic dermatitis could be termed as 'seborrhiasis'[6].

\section{Association with HLA}

A study in Han Chinese patients found that scalp involvement was significantly more prevalent in HLA-Cw6 negative patients than those positive for HLA-Cw6, which differs from the situation in Caucasian patients [1].

\section{Exacerbating factors}

1. Anti-Tumour Necrosis Factor alpha (TNF- $\alpha$ ) agents are commonly used to treat psoriasis but reports have documented newonset or flaring of psoriasis in adults treated for the other conditions. Paediatric cases of anti-TNF- $\alpha$-induced psoriasis presenting with severe scalp involvement are reported [13].

2. It is possible that allergy to particular shampoo, hair styling product or hair dye could exacerbate existing scalp psoriasis [14].

\section{Immunological factors}

There could be association between immunological factors and the development of psoriasis. This has been supported by studies that have found long-term remission from scalp psoriasis following surgical removal of immunological constituents within the dermis [6].

\section{Other causes}

Scalp psoriasis may be caused by an increase in the recruitment of stem cells, resulting in a switch on entry in the anagen phase. Monocytes and macrophages could have a key role in this cascade of events in psoriasis by upregulation of the catenin pathway.

\section{Grading of Scalp Psoriasis}

The 2009 European consensus statement on scalp psoriasis management has defined scalp psoriasis as mild, moderate or severe, based on extent of scalp involvement and severity of lesions (based on the presence and severity of erythema, scaling, pruritus, and thickness) [1]. Grading of scalp psoriasis is mentioned in Table 1. 
Page 2 of 9

\begin{tabular}{|c|c|c|}
\hline Area of involvement & Severity & Morphology, pruritis and thickness \\
\hline$<50 \%$ of the scalp & Mild & $\begin{array}{l}\text { Mild erythema, scaling and pruritus } \\
\text { Minimal thickness }\end{array}$ \\
\hline$<50 \%$ of the scalp & Moderate & $\begin{array}{l}\text { Moderate erythema, and scaling } \\
\text { Mild to moderate pruritus } \\
\text { Moderate thickness }\end{array}$ \\
\hline Affects $>50 \%$ of the scalp & Severe & $\begin{array}{l}\text { Severe erythema and scaling } \\
\text { Moderate to severe pruritus } \\
\text { Hair loss with scarring } \\
\text { Lesions not limited to the scalp (seen on hairline or forehead) }\end{array}$ \\
\hline
\end{tabular}

Table 1: Grading of Scalp Psoriasis

\section{Clinical Features}

\section{In adults}

In psoriasis patients, the scalp is frequently the first site to be affected and often remains affected constantly to some degree over many years, whereas lesions that may have developed subsequently in other sites may remit [6].

Many psoriasis patients present with scalp involvement as initio, even in infancy where "cradle cap" may be a marker for the later development of psoriasis [7]. Typical scalp lesions are well-demarcated and may have thick grey or white scale and patients complain of pruritus and shedding of scales [2].They may occur in the presence or absence of other forms of psoriasis.

Scalp psoriasis may have many phenotypical variants. It is frequently asymmetric due to Koebnerization due to scratching, picking, scrubbing, harsh shampooing, etc. Disease severity on the scalp can be highly variable ranging from mild scaling and erythema with minimal induration to more inflammatory crusted plaque-type forms and even tinea amiantacea with severe crusting enveloping the proximal hair shafts. Areas adjacent to the scalp (forehead, temples, ears and nape of neck) are also frequently involved either by direct extension from the scalp or independently [7].

\section{Severity of scalp psoriasis}

Van Onselen described three severities [14]

1. Mild: Dry flaking skin on areas of the scalp interspersed with normal skin, with unaffected hairline and no hair loss.

2. Moderate: Dry flaking and scaling skin on most of the scalp with some normal skin with extension to hairline and minimal hair loss.

3. Severe: Affection of the entire scalp with minimal normal skin. There are thick and lumpy scales. The hairline is affected, with erythema and scaling extending beyond the scalp margins. Temporary hair loss may occur.

\section{Hair loss in scalp psoriasis}

Scalp psoriasis does not generally result in hair loss, but some increased shedding of telogen hairs and reduction in hair density is common. Also extensive hair loss can occur in the erythrodermic psoriasis and chronic severe hyperkeratotic scalp psoriasis may induce scarring alopecia [6].

\section{In children}

Scalp lesions are present in $47 \%$ to $53 \%$ of children with psoriasis. In fact, the scalp (hairline and occipital scalp) is often the first site of disease involvement in children. In juvenile plaque psoriasis, the plaques are erythematous, with a silvery scale that are finer than in adult and they are typically localized to the scalp, postauricular region, elbows, and knees [9]. At times pityriasis amiantacea, may be the first sign of psoriasis in children and young adults [6].

\section{Scalp psoriasis and hair loss (psoriatic alopecia)}

Shuster first described psoriatic alopecia and till date less than 60 cases of psoriatic alopecia are reported. It was initially thought to be associated exclusively with acute erythrodermic, generalized pustular psoriasis or chronic plaque psoriasis.It is not known whether the alopecia in psoriasis is a scarring or non-scarring process. Some histopathologic studies indicate a scarring process (i.e., reduction in hair follicle density and presence of a peri-infundibular lymphocytic infiltrate with destruction of the follicle).But clinical complete hair regrowth with topical anti-psoriatic treatments favour a non-scarring process.

An alternate explanation relates to the perifollicular inflammation of the upper "permanent" portion of the hair follicle present in psoriatic alopecia (common to all scarring alopecias). Near this site (particularly where the arrector pili attaches), the bulge contains stem cells that give rise to multipotent matrix cells. It is thought that these multipotent cells give rise to the hair shaft, sebaceous gland and adjacent epidermis. Thus, damage to this region compromises the sebaceous gland [15].

\section{Three types of psoriatic alopecia include}

1. Localized: Hair loss confined to lesional skin as confirmed by hair pluck revealing dystrophic bulbs (most common).

Histopathology: Similar to non-scarring alopecia with specific finding of sebaceous gland atrophy.

2. Acute diffuse hair fall: Acute hair fall with a predominance of telogen hairs.

Histopathology: Perifollicular infiltrate and increase in telogen hair. 
3. Destructive alopecia (scarring type): Destructive or scarring alopecia associated with decreased hair density and "perifollicular inflammation with destructional folliculitis and fibrous tissue replacement" (least common).

Histopathology: Similar to other scarring alopecia.

\section{Dermatoscopic findings in scalp psoriasis}

The well-known dermoscopic criteria of psoriasis can also be seen in lesions located on the scalp [16]. G-W. Kim showed that the evaluation of vascular patterns by dermoscopy can be valuable for the clinical diagnosis and differentiation of scalp psoriasis and seborrhoeic dermatitis.According to them the most significant dermoscopic features of scalp psoriasis were red dots and globules, twisted red loops, and glomerular vessels. Seborrhoeic dermatitis was characterized by arborizing vessels and atypical red vessels and absence of red dots and globules. Areas devoid of any particular vascular patterns were also frequently observed in seborrhoeic dermatitis [17].

Melike Kibar in their study found atypical red vessels, Red Dots and Globules (RDG), Signet Ring Vessels (SRV), structure less red areas and Hidden Hairs $(\mathrm{HH})$ statistically more common in psoriasis while twisted red loops and Comma Vessels (CV) in seborrheic dermatitis [18].

\section{Atomic force microscopy (AFM)}

The hair shafts of patients with scalp psoriasis show macropits using Atomic Force Microscopy (AFM) supporting the generalized nature of psoriasis, with changes in hair being analogous to the changes in skin and nails [19].

\section{Diagnosis}

Diagnosis of scalp psoriasis is mainly clinical with features of the dry flaky scalp and well demarcated plaques.

A personal or family history of psoriasis or confirmed or suspected psoriasis on other body parts further supports the diagnosis. Skin biopsy can confirm diagnosis in uncertain cases, however there is no blood or laboratory test for scalp psoriasis [14].

\section{Histology of scalp psoriasis}

Features include classical epidermal changes of psoriasis and follicular-related changes like infundibular dilatation, perifollicular inflammation and fibrosis, thinned follicular epithelium and fibrous tracts [15]. Hallmark of scalp psoriasis is regression of the sebaceous glands and penetration of lymphocytes and polymorphonuclear leucocytes into the epidermis $[6,20]$. One possible explanation for sebaceous gland atrophy is that psoriasis has an extremely complex cytokine milieu. The psoriatic plaque is characterized by the predominance of cytokines produced by TH1 cells, these include IFN$\gamma$, IL-2, and TNF- $\alpha$. The interactions of cytokines and chemokines produced in lesional epidermis could cause sebaceous glands to atrophy in an autocrine and paracrine manner [15] (Figure 1).

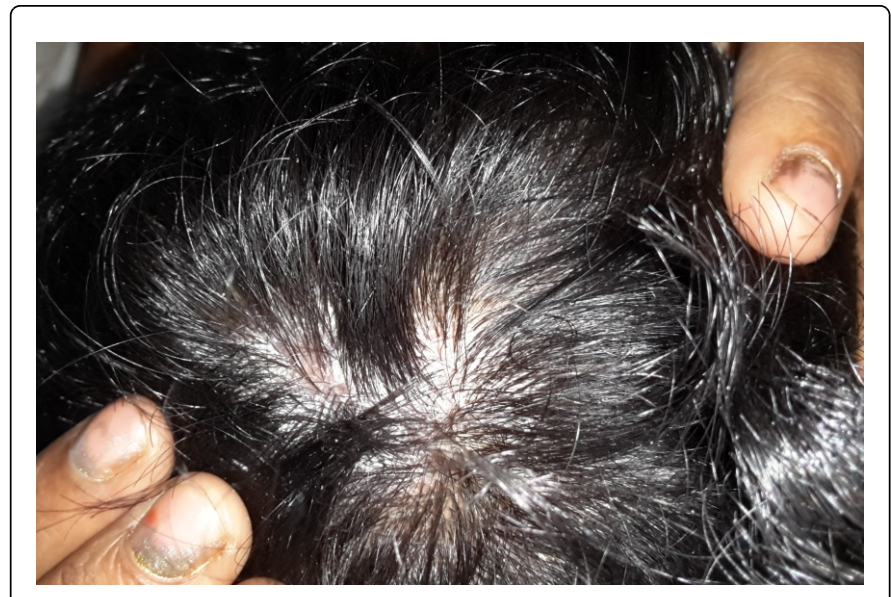

Figure 1: Scaling in scalp psoriasis.

\section{Quality of life in patients with scalp psoriasis}

According to Zampieron et al scalp psoriasis patients suffer from a lower QoL relating to the highly visible sites of their psoriatic lesions $[1,21]$, therefore long term treatment is needed in most patients [3]. Within the adolescent age group, quality of life may be significantly compromised, with impairment of social development especially when alopecia develops in severe cases [5,9]. DLQI is not always sensitive enough to detect low mood and depression and it is important to encourage patients to talk about their wellbeing in addition to use of the DLQI [14] (Figure 2).

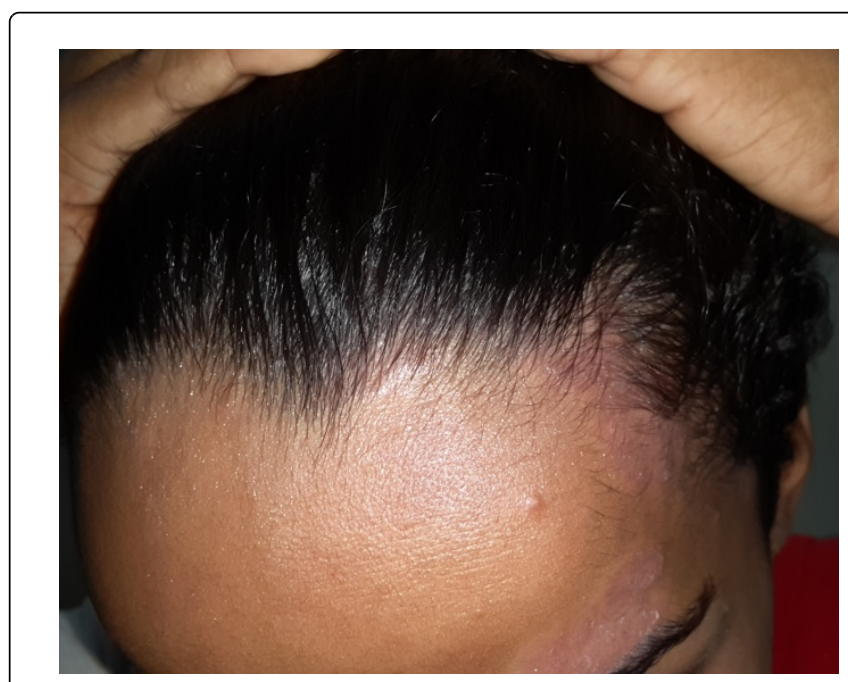

Figure 2: Thin psoriatic plaques on the left side of hairline.

\section{QoL assessment instrument for scalp psoriasis}

a. Scalpdex: The psoriasis scalp index (Scalpdex) is an instrument designed specifically for quality-of-life issues related to scalp dermatoses and can be utilized longitudinally to measure the impact of scalp psoriasis. It is a useful tool to evaluate and monitor the impact on quality of life of scalp psoriasis, in a sustained way throughout the treatment regimen $[7,8]$. It is a validated questionnaire consisting of 23 
questions, taking 5-10 min for completion. Patients use a 5-point Likert scale to evaluate the impact of their scalp condition on each QoL parameter, as follows: $0=$ "never," $1=$ "rarely," $2=$ "sometimes," $3=$ "often," and 4="all the time." [1].

The Children's Scalpdex in Psoriasis (CSP) proves to be reliable, responsive and valid for the assessment of QoL of children with scalp psoriasis. The CSP can be used as a targeted questionnaire in the evaluation of outcome assessments and the effect of therapeutic interventions on QoL in children with scalp psoriasis [8].

b. Scalp psoriasis severity index: Psoriasis Area and Severity Index (PASI) is used to determine psoriasis severity. But this measure is weighted according to the percentage body surface area of involvement; hence it may underestimate the severity of scalp psoriasis if there are few lesions on other surfaces. Hence a modified version of the PASI has been developed specifically for scalp psoriasis called the Psoriasis Scalp Severity Index (PSSI). But PSSI is not used routinely in clinical practice, but it may have value in clinical trials [1].

c. Physician global assessment (PGA):It substantially redundant and sufficient tool for assessing psoriasis severity in patients with moderate to severe disease [22].

\section{d. Patients global assessment}

Treatment principles and guidelines: Scalp psoriasis presents unique challenges for treatment and few guidelines are specific to the management. The scalp is rendered relatively inaccessible to topical therapies or ultraviolet (UV) light due to presence of hair. Also scalp and facial skin may be more susceptible to the irritant effects of treatment.

The Asia Scalp Psoriasis Study Group (ASPSG) advocates a patientcentered approach to treatment, since patients with scalp psoriasis may have only a small proportion of their total body surface area affected, but the effects of social isolation and other QoL issues may be profound.The rationale for this approach is to facilitate treatment decisions that take account of both the objective and subjective severity of scalp psoriasis. Treating physicians should consider patient QoL, prior treatment response, formulation preferences, likely adherence, cost, time available for self-management, and potential adverse events [1].

Older treatments: These include dermatome shaving, phototherapy, pulsating magnetic fields and Grenz rays.One of the first topical preparations was a mixture of liquid petrolatum, sodium chloride solution and phenol [6].

Current treatments: The mainstay of treatment for scalp psoriasis is topical treatment which is recommended as first-line therapy for all patients. The choice of treatment should be determined by disease severity, patient preference, previous response and cost. The vehicle and active ingredients are relevant to efficacy, tolerability and compliance [1].

Vehicle: The vehicles used can be either rinsed off as a shampoo or applied directly to the scalp (e.g. in the form of an alcohol-containing lotion, gel, foam, emulsion, cream, ointment or oil).

Active ingredients: Active ingredients include keratolytics, coal tar, dithranol, retinoids, antifungals, corticosteroids and vitamin D3 analogues $[1,6]$.

First line therapy $[23,24]$ :
- Topical corticosteroids

- Vitamin D 3 analogues

- Salicylic acid/Urea

- Calcipotriol

- Dithranol/Anthralin

- Coal tar

- Tazarotene

- Combination therapies

Second-line therapy:

- Phototherapy

- Systemic therapies (Methotrexate, Acitretin, Cyclosporine)

- Biologics

\section{Products for Scalp [24]}

\section{Leave on products}

These include gels, lotions and ointments containing steroids, coal tar, salicylic acid or vitamin D analogs.

\section{Wash off products}

These include shampoos containing coal tar, salicylic acid, sulfur, selenium, ketoconazole or zinc pyrithione.

\section{Phases of treatment [24]}

Scalp psoriasis treatment has been divided into four phases by Van de Kerkhof et al.

1. First phase: Descaling using salicylic acid or urea preparations.

2. Second phase: Clearing phase in which topical corticosteroids, vitamin $\mathrm{D}$ analogs, tar, dithranol, antifungals, ultraviolet light or systemic treatments are used.

3. Third phase: Stabilization using a steroid sparing vitamin D analog during the week and a super potent topical corticosteroid at weekends.

4. Fourth phase: Maintenance phase, using a vitamin D analog alone or with a tar shampoo.

\section{Topical treatments for scalp psoriasis}

\section{A. Topical corticosteroids}

They have anti-inflammatory and anti-proliferative, properties reducing pruritus, scaling and erythema [9].Advantage of steroids is rapid action, limited adverse effects and patient friendly formulations (can be administered by means of any vehicle) $[4,6]$.

Formulations: Short contact formulations such as shampoos, lotions, foams and gels are preferred for patients with mild to moderate disease. Occlusive formulations such as creams and ointments are used for moderate to severe disease.

Side effects: Potent topical steroids can cause skin atrophy, telangiectasia, striae when used long-term, but these are rare on the scalp, because scalp skin is relatively thick and steroid absorption is limited by the presence of hair and sebum. 
Duration of use of steroids: No studies are available to support the safety of the topical corticosteroid effect on the scalp after 4 weeks. The lowest strength preparation for lesion clearance should be used, considering that high-potency steroids may be needed for moderate to severe disease.Long-term use of mid-potency preparations or intermittent use of potent steroids alternating with vitamin $\mathrm{D} 3$ derivatives either combined or not with shampoo containing liquor carbonis detergens is the most suitable treatment for most patients, as there is chance of hypothalamic-pituitary-adrenal axis suppression associated with long-term use of corticosteroids $[1,23]$.

Combinations of topical steroids: Topical steroids with PUVASOL give better results than PUVASOL used alone. Tazarotene has also been found to be efficacious in combination with topical steroids and calcipotriol [24].

Newer vehicles for topical steroids: Foam vehicles are the new alternatives to traditional topical preparations. They have the advantage of minimal residue, increased ease of application, rapid absorption, higher bioavailability, once-daily administration, better compliance and no association with suppression of the hypothalamic pituitary adrenal (HPA) axis. Examples of foams include CP foam $0.05 \%$ and Betamethasone Valerate (BMV) thermolabile, low-residue foam $0.12 \%$ [24].

\section{B. Topical vitamin $D$ analogs}

Vitamin D3 analogues include calcipotriol, maxacalcitol, tacalcitol and calcitriol available since 1992. They inhibit epidermal proliferation, enhance normal keratinization and inhibit inflammation and thus have a substantial anti-psoriatic effect [6].

Formulations: Vitamin D3 analogs, most commonly calcipotriol (also called calcipotriene), are also available in solution, lotion or gel formulations. Lotion formulations are a patient friendly, tolerable and effective alternative to corticosteroids [1].They are also available in water- and oil-based scalp formulations [23].

Side effects: They are only minimally systemically absorbed and therefore have few systemic side effects and are not associated with atrophy and may be used for long-term, however irritation (temporary) occurs during the first few weeks of treatment. Other side effects include burning, itching, irritation and dryness. Calcipotriol is the preferred vitamin D3 analog because it has far less effect (by a factor of 100 - to 200-fold) than calcitriol on systemic calcium metabolism, resulting in a better safety profile $[1,23,24]$.

Duration of treatment: The $0.005 \%$ solution is applied to the affected area and rubbed gently into the scalp twice daily. Response to therapy takes about 8 weeks, in contrast to $2-3$ weeks for topical corticosteroids.

Contraindications [24]: Acute psoriatic eruptions of the scalp, Hypercalcemia, Hypervitaminosis D

Combinations: Combination of Vitamin D 3 analogues with topical steroids are associated with lesser side effects, greater antiinflammatory and antiproliferative effects, better tolerance, marked impact on QoL, use of lower doses of each used and utility as maintenance therapy. Calcipotriol plus betamethasone dipropionate gel is a well-tolerated and effective once-daily treatment regimen for scalp psoriasis vulgaris in adolescents [1,5].

\section{Keratolytics}

For scaling associated with moderate or severe scalp psoriasis keratolytics, such as salicylic acid are beneficial, prior to initiating other forms of topical therapy (many topical treatments do not work well until the thick scales, which reduce the drug penetration, are removed) [1]. Salicylic acid, 5 and $10 \%$, is the most widely used keratolytic agent, and is formulated in a solution, gel, ointment or petroleum jelly [23].

\section{Anthralin}

Anthralin $0.1-3 \%$ cream has been used for long-term treatment of scalp psoriasis. It is applied in a thin layer to the psoriatic area once daily, rubbed in well and left on the scalp for 5-10 minutes before washing with shampoo and rinsing. Cream concentration should be gradually increased according to body response and patient tolerance. It may also be employed safely in combination with other antipsoriatic medications. It is not used for acutely inflamed scalp psoriasis. Side effects include redness, irritation, temporarily staining of fingernails, gray/white hair, skin and fabrics $[24,25]$.

\section{E. Coal tar preparations}

Crude coal tar is the most effective tar available for the treatment of scalp psoriasis with a potent efficacy against pruritus. On the scalp, the application of crude coal tar is difficult, hence coal tar shampoos, containing 2 to $10 \%$ coal tar solution are effective. Topical tar solution [liquor picis carbonis (LPC) or liquor carbonis detergens (LCD)] is widely available and commonly used for scalp psoriasis. Coal tar preparations are inexpensive and suitable treatments for scalp psoriasis. They are used twice weekly.Disadvantages include unpleasant odor, staining properties, messy application and mutagenic potential $[1,3,6,24]$.

Newer preparations for scalp include coconut oil compound ointment (coal tar solution with precipitated sulfur, salicylic acid, coconut oil, yellow soft paraffin and emulsifying wax) and tar pomades (LCD, Tween 20 and salicylic acid in a hydrophilic ointment). Compound ointment is applied once at night and washed off in the morning using coal tar shampoo [24].

\section{F. Dithranol}

Dithranol is an older form of treatment for psoriasis, though now rarely used being reserved for more severe and treatment-resistant cases. Disadvantages include burning sensation (reducing patients acceptability), staining the skin or hair, and inability to be washed out easily $[1,24]$.

\section{G. Antifungal agents}

Antifungal agents may have a limited role in the treatment of scalp psoriasis, based on the etiological role of overgrowth of Malassezia species (plays role in development of both scalp psoriasis and seborrheic dermatitis), but their efficacy in treatment of scalp psoriasis is inconsistent.

Ketoconazole has anti-inflammatory effects and antifungal activity and may be useful for mild psoriasis. Antifungals are probably most suitable for sebopsoriasis patients or immunocompromised patients. In resistant cases, topical imidazole derivatives are used to control the overgrowth of Pityrosporum [24]. The combination of bifonazole $1 \%$ 
Page 6 of 9

with urea $40 \%$ has been reported to be effective in scalp psoriasis and seborrhoeic dermatitis [6].

\section{H. Tazarotene}

There are no controlled studies for tazarotene $(0.1 \%)$ use in scalp psoriasis. The response is less as compared to topical calcipotriol or steroids, but relapse rates are less. Dryness and irritation are the common side effects [24].

I. Moisturizing: Moisturizing can reduce itching, skin tightness and discomfort, cause scale softening and gentle lifting of plaques. It can increase effectiveness of topical treatments with active ingredients.Since the scalp is a predominantly hairy area, emollients should be thinner and less greasy than those used on other parts of the body. Lotions and oils are most appropriate and they should be applied before bed, and washed out in the morning to minimize any unpleasant cosmetic effects. Recommended oils are coconut, olive or arachis oil, applied at night under occlusion [14]. Urea 10\% and lactic acid $10 \%$ have been used as scalp moisturizers [24]. Table 2 mentions the various topical treatments with their action.

\begin{tabular}{|l|l|}
\hline Topical treatment & Action \\
\hline Topical steroids & Helpful on localized lesions and requires monitoring \\
\hline Vitamin D analogues & $\begin{array}{l}\text { Is cosmetically acceptable. } \\
\text { Contraindicated in pregnancy and breastfeeding. } \\
\text { Can be combined with corticosteroid ointments for short-term use. }\end{array}$ \\
\hline Keratolytics & Can help reduce excessive scaling, but may irritate surrounding skin. \\
\hline $\begin{array}{l}\text { Dithranol (including short contact } \\
\text { therapy) }\end{array}$ & Good for treating chronic scaly psoriasis in selected areas. \\
\hline $\begin{array}{l}\text { Vitamin A analogues } \\
\text { Tar preparations }\end{array}$ & $\begin{array}{l}\text { To be applied once daily. } \\
\text { Not recommended on face, skin folds or large areas of the body. } \\
\text { Contraindicated in pregnancy and breastfeeding. }\end{array}$ \\
\hline $\begin{array}{l}\text { Emollients } \\
\text { (moisturizers) }\end{array}$ & $\begin{array}{l}\text { Help to remove loose scales. } \\
\text { Are messy and stains clothing. }\end{array}$ \\
\hline
\end{tabular}

Table 2: Topical treatment of scalp psoriasis [14].

\section{Treatment Ladder}

It is recommended that treatment should begin with a potent corticosteroid once daily. If no adequate improvement is seen in four weeks, a different potent corticosteroid formulation should be used, with addition of a descaling agent such as salicyclic acid. If the response continues to be inadequate, a vitamin $\mathrm{D}$ and corticosteroid combination, or vitamin $\mathrm{D}$ alone should be given [14].

If the response is inadequate after eight weeks of this therapy, coal tar preparation can be used (Figure 3).

\section{Cosmetic and general care [14]}

1. Regular hair washes to remove debris of medication

2. Demonstration of application: Emollients and non-shampoo topical treatments application involves parting the hair in sections and rubbing the treatment along the exposed area in a sequential fashion.

3. Education: Patient should be informed that commonly available hair care products have no negative effect on scalp psoriasis or adverse interaction with psoriasis medications. 


\section{Potent corticosteroid: use once daily for up to four weeks}

If response is not adequate
within four weeks

Different corticosteroid formulation and/or descaling agent

If response is not adequate
within four weeks

Calcipotriol and betamethasone combination treatment once daily or vitamin $\mathrm{D}$ once daily

If response is not adequate

Very potent corticosteroid use up to twice daily for two weeks or coal tar once

or twice daily

Figure 3: Algorithm for scalp psoriasis treatment.

\section{Limitations of topical therapy}

Although there is a broad range of topical therapies, certain factors that can limit treatment options, these include

Negative effects on hair quality causing oiliness, dryness, discoloration, irritation, unpleasant odour, convenience, ease of application, cosmetic acceptability, effectiveness for reducing itch and scale, safety for prolonged use without loss of benefit.

These factors may lead to increased itching and scratching, which in turn may precipitate a trauma-induced flare-up (Koebner phenomenon) $[23,26]$.

\section{Systemic therapies}

Systemic therapies, such as methotrexate, acitretin, cyclosporine or biologic agents should be reserved for step two therapy, in patients with severe and/or treatment-resistant scalp psoriasis, or in patients with pre-existing moderate to severe cutaneous psoriasis or psoriatic arthropathy [1]. Patients with mild psoriasis who have significant scalp involvement may be candidates for systemic treatment because involvement of large areas of the scalp can justify reclassification of psoriasis as moderate to severe [26].

\section{Various systemic treatments}

A. Light and laser therapy: Presence of hair on scalp can make delivery of UV rays to the scalp difficult. Phototherapy tends to be poorly effective on unshaven scalp, is not universally available and requires patients to attend clinics for treatment. Therefore, phototherapy should be reserved for severe or treatment-resistant scalp psoriasis in patients who have access to this treatment. Better results are achieved with conventional UV units, if hair is parted in many rows or if the patient has thin hair or if the head is shaved. A UVB comb has been successfully used to deliver UV rays directly to affected scalp (as per data from small uncontrolled studies). Excimer laser therapy appears to be more effective than narrow-band UVB therapy or pulsed-dye laser therapy. A hair blower can be used to part the hair prior to use of a $308 \mathrm{~nm}$ excimer laser. Also excimer laser can be combined with topical psoralen and UVA irradiation (PUVA) to speed healing and reduce the cumulative UVA dose relative to PUVA alone $[1,6]$.

The excimer laser is a form of narrowband ultraviolet B (UVB) phototherapy delivering $308 \mathrm{~nm}$ radiation but its high output makes it technically possible to treat individual areas of psoriasis in short periods of time. This therapeutic advantage, combined with some type of mechanical (manual) separation of the hair, appears to effectively treat scalp psoriasis.Furthermore, when used in a supra erythemogenic mode involving exposure of plaques to several multiples of the minimal erythema dose rapid clearance can be achieved followed by prolonged remissions. Disadvantages is that it is time consuming requiring multiple sessions and maintenance treatment is needed in order to maintain the results leading to low patient compliance $[23,24,27]$. 
Page 8 of 9

B. Grenz rays: Grenz ray therapy, involves use of electromagnetic radiation similar to X-rays, but with reduced penetration.It has been shown to be an effective and convenient alternative treatment for scalp psoriasis when topical agents have failed [6].

C. Biologics: Biological agents which block tumour necrosis factor such as etanercept,infliximab and adalimumab have the longest established clinical experience;however, their use in scalp psoriasis is based on low-level evidence [23].

Adalimumab: Patients with scalp involvement may be candidates for systemic treatment even if they are classified as having mild disease. Adalimumab may be beneficial for scalp psoriasis, even in patients who have failed other systemic therapies [26].

Alefacept: Alefacept is a recombinant, fully human fusion protein consisting of domains of leukocyte-function associated antigen type 3 and immunoglobulin G. It selectively reduces the number of memory $\mathrm{T}$ lymphocytes, important mediators of pathogenesis in psoriasis, by inhibiting their proliferation and inducing apoptosis.James et al showed that alefacept is effective in a subset of patients with scalp psoriasis, is well tolerated and is a good treatment option for patients with widespread scalp psoriasis [28].

Etanercept:Etanercept has been found to be effective and welltolerated for scalp psoriasis, showing a statistically significant difference in the PSSI between the experimental and control groups in one study [23].

Ixekizumab (IXE): Ixekizumab (IXE) is a monoclonal antibody to IL-17A, a cytokine in psoriasis pathogenesis. In the UNCOVER trial, $60 \%$ of IXE-treated patients achieved complete resolution of their scalp psoriasis by week 12 , which was significantly more than those treated with either ETN or PBO [29].

Ustekinumab: Ustekinumab is a human monoclonal antibody that binds to the p40 subunit common to both interleukins 12 and 23, produces rapid and durable improvement in moderate to severe plaque psoriasis with improvement in the quality of life. At present, ustekinumab treatment is a second-line therapy for patients with severe refractory scalp psoriasis [23].

D. Methotrexate: Methotrexate was found to be effective in scalp psoriasis with an optimal therapeutic effect reaching after 2-3 months, but no studies have specifically evaluated its effect in scalp psoriasis [23].

E. Retinoids: Acitretin has no specific use in the treatment of scalp lesions. Positive results are usually observed only after 24 weeks of treatment, its limitation being causation of hair loss [23].

F. Apremilast: Cyclic adenosine monophosphate is a key modulator of immune cell responses, and its levels are regulated by phosphodiesterase. Apremilast, an oral phosphodiesterase 4 inhibitor, increases intracellular cyclic adenosine monophosphate levels, thus regulating production of proinflammatory and anti-inflammatory mediators. Apremilast was approved by the US Food and Drug Administration in 2014 and by the European Commission in 2015 for active psoriatic arthritis and moderate to severe plaque psoriasis. The ESTEEM study demonstrated that Apremilast $30 \mathrm{mg}$ BID significantly improves scalp psoriasis with sustained improvement over time [30].

\section{Treatment compliance [9]}

Psoriasis treatments are often associated with poor patient adherence. Common reasons for poor adherence are longer duration of treatment, frequency of administration, type of formulation, efficacy of the drug, perception of safety of the drug, physician-patient relationship, and quality of the vehicle.

Treatment failures are often a result of failure to apply the medication rather than failure of the medication itself. Nurses can play an important role in educating patients about the importance of adherence and in providing instructions on how to apply, how much to apply and for how long to apply. This is particularly important with vehicles such as foams which patients may not know how to use [7].

\section{Prognostic implication of scalp psoriasis}

The scalp psoriasis is likely to be less associated to psoriatic arthropathy, but may be an important comorbidity factor for the development of psoriatic arthropathy [31].

\section{Conclusions}

Around $75 \%$ and $90 \%$ of Asian patients with psoriasis has scalp involvement. A patient-centered approach to treatment should be done, since patients with scalp psoriasis may have only a small proportion of their total body surface area affected, but the effects of social isolation and other QoL issues may be profound. More pervasive use of the Scalpdex instrument is recommended. Treatment should be individualized and based on objective severity of the disease. Topical therapy is the mainstay of treatment and factors like effectiveness, speed of response, patient preference, adherence, cost, time available for self-management and potential adverse events should be considered while choosing topical therapy. Oral therapy should be reserved for severe or treatment resistant scalp psoriasis. Patient education about use of medications, expectations of efficacy and tolerability issues and long-term management options will help achieve adherence and therapeutic success [1].

\section{References}

1. Frez ML, Asawanonda P, Gunasekara C, Koh C, Loo S, et al. (2014) Recommendations for a patient-centered approach to the assessment and treatment of scalp psoriasis: a consensus statement from the Asia Scalp Psoriasis Study Group. J Dermatolog Treat 25: 38-45.

2. Crowley J (2010) Scalp psoriasis: an overview of the disease and available therapies. J Drugs Dermatol 9: 912-918.

3. van de Kerkhof PC, Franssen ME (2001) Psoriasis of the scalp. Diagnosis and management. Am J Clin Dermatol 2: 159-165.

4. van der Vleuten CJ, van de Kerkhof PC ( 2001) Management of scalp psoriasis: guidelines for corticosteroid use in combination treatment. Drugs 61: 1593-1598.

5. Gooderham M, Debarre JM, Grant JK, Xu Z, Kurvits M, et al. (2014) Safety and efficacy of calcipotriol plus betamethasone dipropionate gel in the treatment of scalp psoriasis in adolescents 12-17 years of age. Br J Dermatol 171: 1470-1477.

6. Papp K, Jones JB, Kragballe K, Wozel G, de la Brassinn M (2007) Scalp psoriasis: a review of current topical treatment options. J Eur Acad Dermatol Venereol 21: 1151-1160

7. Kragballe K, Menter A, Lebwohl M, Tebbey PW, van de Kerkhof PC (2013) Long-term management of scalp psoriasis: perspectives from the International Psoriasis Council. J Dermatolog Treat 24: 188-192. 
8. Oostveen AM, Jong EM, Evers AW, Donders AR, van de Kerkhof PC, et al. (2014) Reliability, responsiveness and validity of Scalpdex in children with scalp psoriasis: the Dutch study. Acta Derm Venereol 94: 198-202.

9. Osier E, Gomez B, Eichenfield LF (2015) Adolescent Scalp Psoriasis: Update on Topical Combination Therapy. J Clin Aesthet Dermatol 8: 43-47.

10. Farr PM, Krause LB, Marks JM, Shuster S (1985) Response of scalp psoriasis to oral ketoconazole. Lancet 2: 921-922.

11. Gomez-Moyano E, Crespo-Erchiga V, Martínez-Pilar L, Godoy Diaz D, Martínez-García S, et al.(2014) Do Malassezia species play a role in exacerbation of scalp psoriasis? J Mycol Med 24: 87-92.

12. Prohić A (2003) Identification of Malassezia species isolated from scalp skin of patients with psoriasis and healthy subjects. Acta Dermatovenerol Croat 11: 10-16.

13. Perman MJ, Lovell DJ, Denson LA, Farrell MK, Lucky AW (2012 ) Five cases of anti-tumor necrosis factor alpha-induced psoriasis presenting with severe scalp involvement in children. Pediatr Dermatol. 29: 454-459.

14. Renton C (2014) Diagnosis and treatment of adults with scalp psoriasis. Nurs Stand 28: 35-39.

15. Silva CY, Brown KL, Kurban AK, Mahalingam M (2012) Psoriatic alopecia - fact or fiction? A clinicohistopathologic reappraisal. Indian J Dermatol Venereol Leprol 78: 611-619.

16. Lallas A, Apalla Z, Argenziano G, Sotiriou E, Di Lernia V, et al. (2014) Dermoscopic pattern of psoriatic lesions on specific body sites. Dermatology 228: 250-254.

17. Kim GW, Jung HJ, Ko HC, Kim MB, Lee WJ, et al. (2011) Dermoscopy can be useful in differentiating scalp psoriasis from seborrhoeic dermatitis British Journal of Dermatology 164: 652-656.

18. Kibar M, Aktan Ş, Bilgin M (2015) Dermoscopic Findings in Scalp Psoriasis and Seborrheic Dermatitis; Two New Signs; Signet Ring Vessel and Hidden Hair. Indian J Dermatol 60: 41-45.

19. Shin MK, Kim KS, Ahn JJ, Kim NI, Park HK, et al. (2012) Investigation of the hair of patients with scalp psoriasis using atomic force microscopy. Clin Exp Dermatol 37: 156-163.

20. Werner B, Brenner FM, Böer A (2008) Histopathologic study of scalp psoriasis: peculiar features including sebaceous gland atrophy. Am J Dermatopathol 30: 93-100.

21. Zampieron A, Buja A, Fusco M, Linder D, Bortune M, et al. (2015) Quality of life in patients with scalp psoriasis. G Ital Dermatol Venereo 150: 309-316.
22. Robinson A, Kardos M, Kimball AB (2012) Physician Global Assessment (PGA) and Psoriasis Area and Severity Index (PASI): why do both? A systematic analysis of randomized controlled trials of biologic agents for moderate to severe plaque psoriasis. J Am Acad Dermatol 66: 369-375.

23. Papadavid E, Ferra D, Koumaki D, Dalamaga M, Stamou C, et al. (2014) Ustekinumab induces fast response and maintenance of very severe refractory scalp psoriasis: results in two Greek patients from the psoriasis hospital-based clinic. Dermatology 228: 107-111.

24. Handa S (2010) Newer trends in the management of psoriasis at difficult to treat locations: Scalp, palmoplantar disease and nails. Indian J Dermatol Venereol Leprol 76: 634-644.

25. Menter A, Dallas TX, Deliduka S, Elmhurst IL, Torok H, et al. (2015) Patient-centered outcomes trial of anthralin microcrystalline encapsulated shampoo for treatment of scalp psoriasis. JAAD 72: AB242

26. Thaçi D, Unnebrink K, Sundaram M, Sood S, Yamaguchi Y (2015 ) Adalimumab for the treatment of moderate to severe psoriasis: subanalysis of effects on scalp and nails in the BELIEVE study. J Eur Acad Dermatol Venereol 29: 353-360.

27. Morison WL, Atkinson DF, Werthman L (2006) Effective treatment of scalp psoriasis using the excimer $(308 \mathrm{~nm})$ laser. Photodermatol Photoimmunol Photomed 22: 181-183.

28. Krell J, Nelson C, Spencer L, Miller S (2008) An open-label study evaluating the efficacy and tolerability of alefacept for the treatment of scalp psoriasis. Journal of the American Academy of Dermatology 58: 609-616.

29. Reich K, Lebwohl M, Romiti R, Sofen H, Goldblum O, et al. (2016) Impact of ixekizumab treatment on scalp psoriasis: Results from the UNCOVER-2 trial. JAAD 74: AB253.

30. Rich P, Gooderham M, Bachelez H, Goncalves J, Day RM, et al. (2016) Apremilast, an oral phosphodiesterase 4 inhibitor, in patients with difficult-to-treat nail and scalp psoriasis: Results of 2 phase III randomized, controlled trials (ESTEEM 1 and ESTEEM 2). J Am Acad Dermatol 74: 134-142.

31. Patrizi A, Venturi M, Scorzoni R, Pazzaglia M, Malavolta N, et al. ( 2014 ) Nail dystrophies, scalp and intergluteal/perianal psoriatic lesions: risk factors for psoriatic arthritis in mild skin psoriasis? G Ital Dermatol Venereol 149: 177-184. 